\title{
Hierarchical Silica Nanostructures Inspired by Diatom Algae Yield Superior Deformability, Toughness, and Strength
}

\begin{abstract}
ANDRE P. GARCIA, DIPANJAN SEN, and MARKUS J. BUEHLER
A universal design paradigm in biology is the use of hierarchies, which is evident in the structure of proteins, cells, tissues, and organisms, as well as outside the material realm in the design of signaling networks in complex organs such as the brain. A fascinating example of a biological structure is that of diatoms, a microscopic mineralized algae that is mainly composed of amorphous silica, which features a hierarchical structure that ranges from the nano- to the macroscale. Here, we use the porous structure found at submicron length scales in diatom algae as a basis to study a bioinspired nanoporous material implemented in crystalline silica. We consider the mechanical performance of two nanoscale levels of hierarchy, studying an array of thin-walled foil silica structures and a hierarchical arrangement of foil elements into a porous silica mesh structure. By comparing their elastic, plastic, and failure mechanisms under tensile deformation, we elucidate the impact of hierarchies and the wall width of constituting silica foils on the mechanical properties, by carrying out a series of large-scale molecular dynamics (MD) simulations with the first principles based reactive force field ReaxFF. We find that by controlling the wall width and by increasing the level of hierarchy of the nanostructure from a foil to a mesh, it is possible to significantly enhance the mechanical response of the material, creating a highly deformable, strong, and extremely tough material that can be stretched in excess of 100 pct strain, in stark contrast to the characteristic brittle performance of bulk silica. We find that concurrent mechanisms of shearing and crack arrest lead to an enhanced toughness and are enabled through the hierarchical assembly of foil elements into a mesh structure, which could not be achieved in foil structures alone. Our results demonstrate that including higher levels of hierarchy are beneficial in improving the mechanical properties and deformability of intrinsically brittle materials. The findings reported here provide insight into general material design approaches that may enable us to transform a brittle material such as silicon or silica into a ductile, yet strong and tough material, solely through alterations of its structural arrangement at the nanoscale.
\end{abstract}

DOI: $10.1007 / \mathrm{s} 11661-010-0477-\mathrm{y}$

(C) The Minerals, Metals \& Materials Society and ASM International 2010

\section{INTRODUCTION}

THE inextricable link between biology and hierarchies is profound. For example, deep sea sponges and diatoms encompass nano- and microlevels of hierarchical silicified structures, which are surprisingly tough and overcome the brittleness of the constituent material. ${ }^{[1,2]}$ The macroscopic structure of the sponge Euplectella sp. is that of a square lattice with diagonal ridges, whereas the lowest levels of hierarchy comprise a cylindrical laminated architecture of silica and protein. Indeed, the hierarchical combination of inorganic with organic materials yields a structure that resists strong sea

ANDRE P. GARCIA, Graduate Student, and MARKUS J. BUEHLER, Associate Professor, are with the Laboratory for Atomistic and Molecular Mechanics, Department of Civil and Environmental Engineering, Massachusetts Institute of Technology, Cambridge, MA 02139. Contact e-mail: mbuehler@mit.edu DIPANJAN SEN, Graduate Student, is with the Laboratory for Atomistic and Molecular Mechanics, Department of Civil and Environmental Engineering, Massachusetts Institute of Technology, and is also with Department of Materials Science and Engineering, Massachusetts Institute of Technology, Cambridge, MA 02139.

Manuscript submitted March 19, 2010.

Article published online January 5, 2011 currents, which impose high bending stresses on the sponge. Although hierarchies within natural materials are observed between vast length scales, the nanoscale is of particular importance when elucidating the fundamental mechanisms that allow for deformation of materials, including elastic, plastic, and fracture properties. ${ }^{[2-4]}$

A fascinating example of biomineralized structures are those of diatoms, a microscopic algae that feature cell walls, or frustules, mainly composed of amorphous silica. ${ }^{[5,6]}$ A nanoporous hierarchical structure dominates the landscape of the cell wall and encompasses intricate patterns that are highly varied and ordered, as shown in Figure 1, and reach all the way down to the nanoscale. Beyond the key biological functions of the cell wall lie important mechanical functions, such as preventing virus penetration, protecting diatoms from the mandibles of predators, or even digestion in some cases. ${ }^{[7-9]}$ Applications of materials and technologies inspired from diatoms are widespread and encompass multiple disciplines. Some examples of applications are filters, molecular sieves, resins, insulators, sensors, electronics, optical coatings, environmental indicators of fresh and salt water, and the reduction of red tide's 


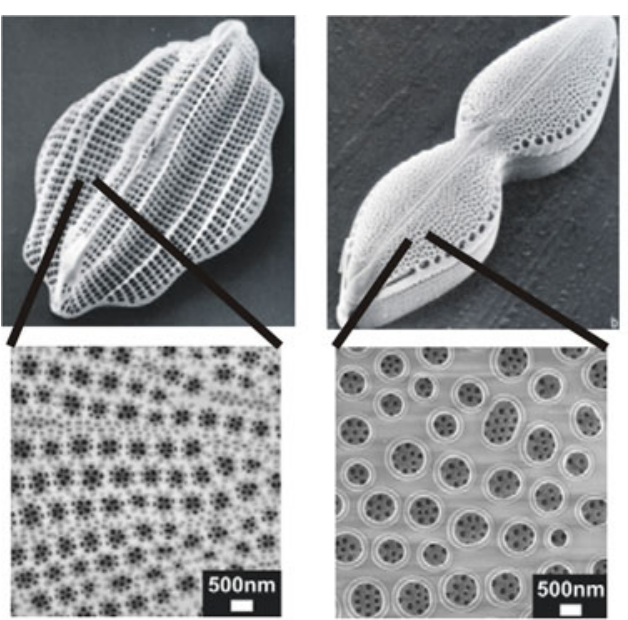

(a)

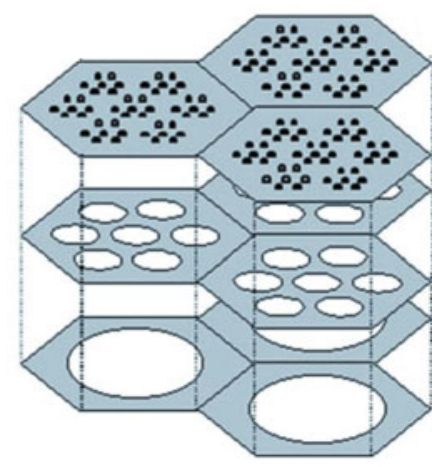

(b)

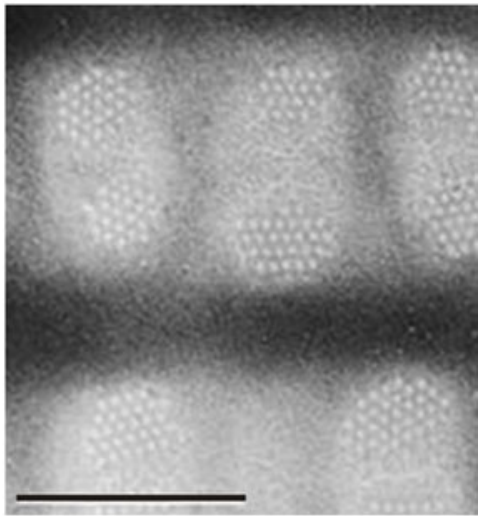

(c)

Fig. 1-Hierarchical structure of diatoms, showing their porous silica structure. (a) SEM images showing various diatom species, displaying the intricate hierarchical porous silica wall structure of diatoms. (b) Panel showing a schematic of the hierarchical structure. (c) Panel showing nanoscale voids observed in diatom algae, which are occluded by a delicate silicate membrane, called a hymen ( $p l$. hymens), which is perforated by round pores (scale bar $200 \mathrm{~nm}$ ). (a) Images are reprinted from Ref. 49 (with permission). (b) Image reprinted from Ref. 13 (with permission). (c) Image reprinted from Ref. 50 (with permission).

toxicity. ${ }^{[10-13]}$ Other novel applications for porous biosilica structures are flexible, high-performance solar cells, and scaffolding systems for cells or a protein matrix. ${ }^{[14,15]}$ From an ecologic perspective, diatoms and blue-green algae in marine environments are perhaps the greatest asset toward sustaining life on earth, since they produce approximately 70 pct of all free oxygen on earth. ${ }^{[16]}$ Furthermore, diatoms are a great source for carbon sequestration, since they absorb nearly a quarter of the earth's $\mathrm{CO}_{2}$ due to photosynthesis and from carrying the carbon to the ocean floor once they die. ${ }^{[17,18]}$

Several studies reported in the recent literature have revealed the mechanical properties of diatom shells. Hamm et al. ${ }^{[19]}$ used a glass needle to load and break diatom frustules in order to probe their mechanical response at failure. Their findings for the $\mathrm{F}$. kerguelensis species revealed an elastic modulus of $22.4 \mathrm{GPa}$ and a maximum stress along the costae of $\approx 0.6 \mathrm{GPa}$ (in tension) and $\approx 0.7 \mathrm{GPa}$ (in compression). Other researchers $^{[20]}$ used atomic force microscopy (AFM) nanoindentation to study the nanoscale material properties of the porous frustule layers of diatoms, identifying pore sizes on the order of several tens of nanometers at the smallest levels in the hierarchy, with ultra-thin silica walls on the order of several nanometers. The pore size ranged from $45 \pm 9,192 \pm 35$, and $1150 \pm 130 \mathrm{~nm}$ for the cribellum, cribrum, and areola layers, respectively. Of the three layers, the cribellum had the lowest hardness and elastic modulus: $0.076 \pm 0.034 \mathrm{GPa}$ and $3.40 \pm 1.35 \mathrm{GPa}$, whereas the areola had the highest: $0.53 \pm 0.13 \mathrm{GPa}$ and $15.61 \pm 5.13 \mathrm{GPa}$, respectively. ${ }^{[20,21]}$ They observed that the variation of mechanical properties between the frustule layers could be influenced by the pore size, pore distance, and porosity, and under different biomineralization processes.

We hypothesize that the nanoporous geometry of the frustules in diatoms is the key to providing enhanced toughness even though the constituting material itself (that is, silica) is inherently brittle. Thereby, the formation of a nanostructured geometry that includes thin, geometrically confined struts of brittle elements may play a crucial role in understanding the material's behavior. Indeed, the effects of geometric confinement on some mechanical properties have been discussed in numerous earlier studies. For example, in a previous study focused on crystalline silicon, ${ }^{[22]}$ we found that by creating an ordered nanoporous silicon structure, the material properties were significantly altered compared to the bulk silicon; specifically, a greater deformability was observed. We found that by varying the widths of the silicon structure, a broad range of toughness could be achieved, with a size-dependent peak in performance.

Other studies of the mechanical properties of brittle materials under extreme geometric confinement, in particular, nanowires, revealed that by decreasing the cross-sectional diameter, the material becomes stronger and is capable of undergoing significantly larger deformation before breaking. ${ }^{[23-25]}$ Experimental studies on silica nanowires of widths ranging from 230 to $800 \mathrm{~nm}$ revealed that fracture stress was influenced by specimen size; however, the modulus was not affected by size. ${ }^{[26]}$ This study also determined that the modulus stayed constant at around $68 \mathrm{GPa}$, whereas the fracture stress varied from 8.77 to $6.35 \mathrm{GPa}$ for the smallest and largest $\mathrm{SiO}_{2}$ wire widths, respectively. Ni et al. ${ }^{[27]}$ experimentally determined that the modulus was independent of diameter for amorphous silica nanowires ranging from 50 to $100 \mathrm{~nm}$ in diameter. On the other hand, Silva et $a l .{ }^{[28]}$ found increasing stiffness for lower diameters by performing molecular dynamics (MD) simulations on amorphous silica nanowires. The fracture toughness of bulk vitreous silica was determined to be approximately $0.8 \mathrm{MPa} \mathrm{m}^{1 / 2}$. ${ }^{[29]}$ The failure mechanisms occurring within the process zone at the crack tip in amorphous silica glass were studied by Bonamy et al. ${ }^{[30]}$ By implementing 


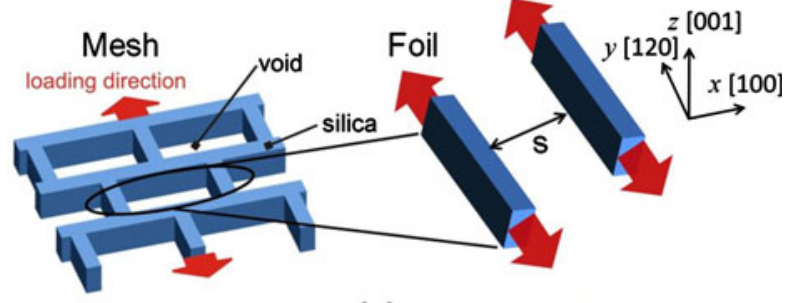

(a)

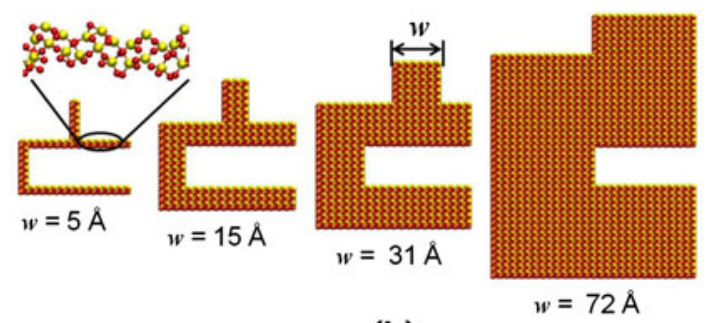

(b)

Fig. 2- Geometry of the bioinspired silica structure and setup used in our simulations. Panel $(a)$ : Three-dimensional schematic of the silica mesh structure (shown on left), with periodic boundaries along the $x, y$, and $z$ directions. On the right, the geometry of the silica foil array structure is shown and has periodic boundaries along the $z$ and $y$ directions with free surfaces only along the $x$-axis. The spacing $s$ between the foils is equal to that in the corresponding mesh structure. The crystallographic orientation is the same for all silica structures considered. The lowest level of hierarchy represented here is the foil, and the highest is the mesh structure. The arrows indicate tensile load applied uniformly along the structure. Panel $(b)$ : Initial geometry of mesh structures considered here, illustrating the wall width ( $w$, definition indicated in one of the structures) variation in the geometry. The inset shows a detailed view of the relaxed surface structure. Wall widths are similarly varied for the foil as in the mesh structure.

AFM experiments and MD simulations, the authors found that nucleation and growth of cavities are the dominant mechanism for failure within the process zone.

Here, we follow the structural framework of diatoms closely, such that it resembles their porous and hierarchical structure, resulting in a similar model system of silica, as shown in Figure 2. We systematically explore two levels of hierarchy in order to ascertain their specific impact in defining the mechanical properties of the structure. First, we consider a foil structure, and second, a mesh structure that is made up of interlocking foils. The reasons for choosing these two levels of hierarchy are as follows: (1) it captures a common structure found in diatoms and other biomineralized materials, (2) it enables us to establish a link between structural geometry and mechanical response, and (3) it provides a general framework to advance future studies of other hierarchical materials. The key question to be addressed is whether the creation of a second hierarchy level yields improvements in material performance, specifically toughness, and maximum stress when compared to the foil structure, or the first hierarchy level. The widths $w$ of the foil and mesh structures are controlled in our analysis and range from 5 to $72 \AA$ (the upper size is limited by computational resources). The approach pursued here is guided by our desire to develop a general model system in which we can test the effect of the size (geometric confinement) of the nanostructure and the level of hierarchy on the bulk material behavior. We use the first principles based ReaxFF reactive force field to describe the interatomic interactions, a powerful model that has been validated against experimental results for mechanical properties, in particular, large deformation and fracture of multiple materials. ${ }^{[31-33]}$ By systematically varying the size and hierarchy of the constituting silica nanostructure, we examine the associated mechanical properties, as well as the fracture and toughening mechanisms, facilitated through a series of MD simulations.

\section{COMPUTATIONAL METHODS}

\section{A. Force Field}

We use a potential based on the ReaxFF force field, which is derived solely from first principles quantum mechanical (QM) calculations. ${ }^{[34]}$ ReaxFF has been shown to accurately describe the behavior of a variety of materials, including bond breaking and the formation process of organics $(\mathrm{C}, \mathrm{O}, \mathrm{H}, \mathrm{N})$, metals $(\mathrm{Cu}, \mathrm{Al}, \mathrm{Mg}$, $\mathrm{Ni}, \mathrm{Pt}$ ), and semiconductors ( $\mathrm{Si}$ ), as well as mixed $\mathrm{Si}-\mathrm{O}$ systems. ${ }^{[23-29]}$ The ability of ReaxFF to describe the large-deformation and bond breaking behavior aspect is critical to describing the properties of materials under large deformation and during failure. Specifically, a well-tested and validated ReaxFF force field is available for silica. ${ }^{[35]}$

\section{B. Model Geometry}

We consider a structural design composed of alphaquartz crystals. One structure is a foil or infinitely tall thin wall, whereas the other is a mesh composed of interlocking silica foils, thus forming uniform and ordered rectangular voids. Since the simulation box is periodic, the foil structure can be thought of as a periodic array of thin foils with a spacing $s$ equivalent to the mesh structure. Figure 2 shows the geometries considered here. Both the mesh and foil structures are exposed to free surfaces. The foil has free surfaces parallel to the $y$-axis, and the mesh has free surfaces along the $x$-axis and $y$-axis. Surface reconstruction may occur and may have some effect on the mechanical behavior. For example, a previous MD study reported a stiffening of silicon nanowires once the surfaces were reconstructed. ${ }^{[36]}$ For a 1.05 -nm-thick nanowire, the modulus increased from approximately 150 to $160 \mathrm{GPa}$ once the [001] surface was reconstructed. The authors attributed this stiffening effect from bond saturation as reconstruction takes place. A previous study used ReaxFF and observed reconstruction of a $\mathrm{ZnO}$ surface after $300 \mathrm{ps}$ at $700 \mathrm{~K}\left(427^{\circ} \mathrm{C}\right) \cdot{ }^{[37]} \mathrm{In}$ another study, it was shown that a total time scale of 240 ps was required to obtain a reconstructed silicon surface by an annealing process with the Tersoff potential and a total system size of 308 atoms. ${ }^{[38]}$ Although it is expected that our ReaxFF based approach can capture surface reconstruction, in principle, it is computationally 
expensive to capture this due to associated time scale and system size, and thus it was not observed in our simulations. Indeed, for small systems such as 1000 atoms, ReaxFF could feasibly simulate the time scales listed previously. However, for systems larger than 10,000 atoms, ReaxFF would be very computationally expensive. The investigation of surface reconstruction and its effects on the mechanics of hierarchical silica structures could be an interesting subject of future studies.

All mesh structures have an equivalent void dimension of 76 by $30 \AA$. The only parameter varied here is the wall width $w$, which ranges from 5 to $72 \AA$ for both foil and mesh (Figure 2). The number of atoms varies from $\approx 750$ to $\approx 17,000$ for the smallest to the largest width silica systems. The largest simulation cell has dimensions of $151 \times 196 \times 8.5 \AA$ in the $x, y$, and $z$ directions. We performed a relaxation for 80 ps of a $31 \AA$ foil structure with free surfaces along the $x$-axis and $y$-axis. It was found that there was negligible average stress after relaxation. This result could be explained since surface reconstruction does not take place.

\section{Simulation Approach}

All structures are equilibrated under the canonical ensemble at $300 \mathrm{~K}$ for a time of $10 \mathrm{ps}$ and then loaded under uniaxial strain loading along the [l $\left.\begin{array}{lll}1 & 2 & 0\end{array}\right]$ direction, as shown in Figure 2(a), at a strain rate of $1 \times 10^{10} \mathrm{~s}^{-1}$ at $300 \mathrm{~K}\left(27^{\circ} \mathrm{C}\right)$. The system has periodic boundary conditions in all three directions and the temperature is controlled by the Berendsen thermostat. ${ }^{[39]}$ Deformation is applied by uniaxially increasing the size of the periodic simulation cell in the loading direction only, while keeping all other dimensions of the simulation cell constant. We use a time-step of 0.2 femtoseconds. The initial, unstrained silica structure is shown in
The engineering strain is defined as

$$
\varepsilon=\frac{\Delta L_{y}}{L_{y}}
$$

where $L_{y}$ is the initial length of the specimen, and $\Delta L_{y}$ is the change in the length along the deformation direction (which is the $y$-coordinate). The stressstrain curve is then used to determine the elastic modulus, $E$, where $\sigma=\sigma_{22}$ (tensile stress in the loading direction):

$$
E=\frac{\partial \sigma}{\partial \varepsilon} \approx \frac{\Delta \sigma}{\Delta \varepsilon}
$$

Once the stress-strain curves are determined, their integral is taken in order to determine the toughness:

$$
E_{V}=\int_{0}^{\varepsilon_{f}} \sigma(\varepsilon) d \varepsilon
$$

where $E_{V}$ and $\varepsilon_{f}$ are the energy per unit volume and strain at failure, respectively. A higher toughness indicates a greater ability of the material to absorb energy due to stresses before failure (resulting, for instance, in a larger fracture process zone). The plastic regime is estimated by measuring the length of the plateau region in the stress-strain curve, which is associated with approximately constant stress beyond the onset of initial yielding. For example, for a silica mesh structure with wall width $w=31 \AA$, plasticity starts at $\approx 47$ pet strain and ends at $\approx 60$ pct strain (the difference in both strains is $\approx 13$ pct, which is what we define as the plastic regime). The measurement is taken from the end of elasticity to the initiation of failure. The Von Mises stress $\sigma_{v}$ is calculated as

$$
\sigma_{v}=\sqrt{\frac{\left(\sigma_{x x}-\sigma_{y y}\right)^{2}+\left(\sigma_{y y}-\sigma_{z z}\right)^{2}+\left(\sigma_{x x}-\sigma_{z z}\right)^{2}+6\left(\sigma_{x y}^{2}-\sigma_{y z}^{2}+\sigma_{z x}^{2}\right)^{2}}{2}}
$$

Figure 2(b). Aside from the variations in the geometry, all simulations are carried out under identical conditions, enabling us to perform a systematic comparison.

\section{Mechanical Analysis}

We calculate the virial stress ${ }^{[40]}$ by

$$
\sigma_{I J}=\frac{\sum_{k}^{N} m_{k} v_{k_{I}} v_{k}}{V}+\frac{\sum_{k}^{N} r_{k_{I}} \cdot f_{k_{J}}}{V}
$$

where $N, m, v, r, f$, and $V$ are the number of atoms, mass of atom, velocity, position, force, and total system volume, respectively. In order to improve image clarity, we only show the stress values associated with silicon atoms when plotting the stress fields. Generally, the oxygen atoms have a much broader stress distribution than silicon atoms, and therefore, the stress patterns are difficult to observe if both atom types are shown. where $\sigma_{I I}(I=x, y, z)$ are the normal components of the stress tensor; and $\sigma_{x y}, \sigma_{y z}$, and $\sigma_{z x}$ are the shear components of the stress tensor. We apply Eq. [5] for the Von Mises stress in plotting the atomic stresses during maximum and failure stresses.

\section{RESULTS AND DISCUSSION}

We begin our analysis by focusing on the effect of changing the wall width on the mechanical properties of the two levels of hierarchy (Figure 2(a) shows the two geometries considered, that is, foil and mesh). As shown in Figure 2, the wall widths are varied between $w=5$ to $72 \AA$ for both the foil and mesh structures. From a structural hierarchy perspective to materials design, the first order of hierarchy consists of a foil oriented such that the only two free surfaces are in the $x$ direction. The second order of hierarchy is composed of a mesh of 
interlocking foils with voids and free surfaces in both $x$ and $y$ directions. The $z$-axis has no free surface, and thus, the structure can be described as consisting of infinitely tall walls or foils.

Figure 3(a) shows the stress-strain response of the foil structure for varying widths $w$, and Figure 3(b) shows the same data for the mesh geometry. Both the foil and mesh structures show an increase of deformation in the plastic regime, a lower modulus, and a lower maximum stress with decreasing wall width $w$. However, the silica mesh structures have a much greater plastic regime than the silica foil. The first important observation made here is that, even though silica is considered a brittle material, the results show that it is possible to transform it into a ductile system for small (nanoscale) wall widths, which reach a maximum failure strain of 90 and 120 pct for the

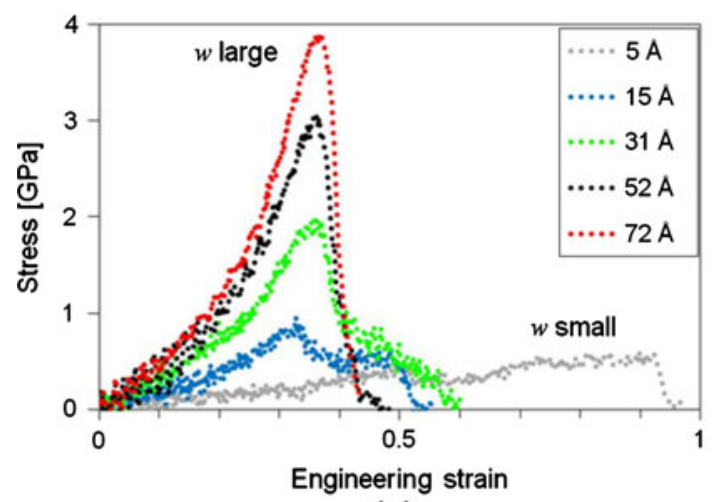

(a)

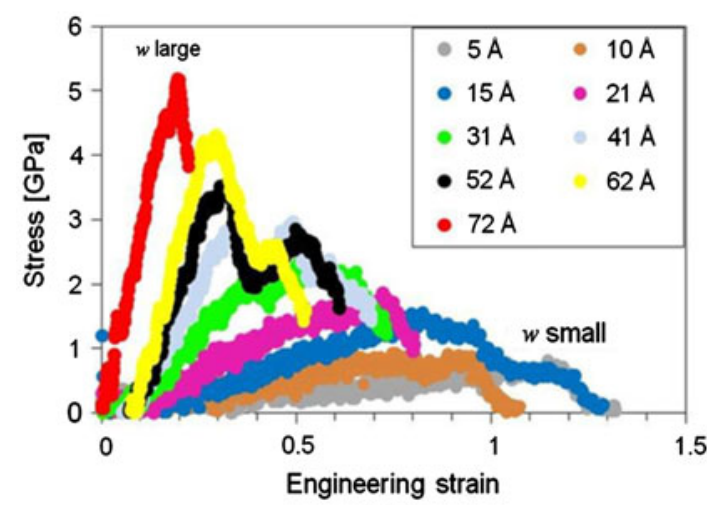

(b)

Fig. 3-Stress-strain graph silica foil (panel (a)) and mesh structure (panel (b)) for all sizes (wall widths range from $w=5$ to $72 \AA$ ). Panel $(a)$ : For smaller wall widths, there is a greater plastic regime, lower maximum stress, and lower modulus. Thus, due to the lowering of the wall width of the structure $(w)$, the system behaves in a more ductile fashion and sustains very large deformation up to 115 pct. The strain hardening region observed for $w=52 \AA$ is due to a temporary crack arrest due to the stretching and shearing of a secondary horizontal ligament. The crack continues its path once the secondary ligament stops deforming. Panel $(b)$ : For wall widths above $15 \AA$, there exists a plastic regime of about 1 to 5 pct. The greatest deformation is obtained for the smallest wall width of $5 \AA$. Failure mechanisms are characterized by void formations near the center or edge of the foil, which then coalesce with other voids until the structure is no longer intact. Cracks are not observed for the foil, whereas in some mesh structures, cracks occur. The reason for stiffening for wall widths $w>15 \AA$ is nonlinear elasticity within the core, along with a Poisson effect, as seen in earlier studies. silica foil and the mesh structure, respectively. In a similar fashion as the foil structures, the silica mesh also shows an increased modulus and maximum stress for larger wall widths. The plastic regime in the mesh structure decreases with increasing wall width, albeit showing a less severe drop than in the case of the foil structure. Interestingly, the maximum tensile stress is reached at roughly the same strain of 34 pct for the silica foil, for all $w \geq 15 \AA$ (Figure 3). Another important difference between the foil and mesh structures lies in a gradual $v s$ a sharp increase of elastic modulus for increasing wall width, respectively. Furthermore, the foil structures show a stiffening effect at widths $w>15 \AA$. Previous studies have also shown either stiffening or softening effects, which are affected by the orientation of the loading (since silica is anisotropic). For example, silica nanorods modeled using the Beest-Kramer-Santen (BKS) and Tsuneyuki-Tsukada-Aoki-Matsui (TTAM) potentials showed a softening effect when under tension. ${ }^{[41,42]}$ Another study conducted ab initio simulations using the projector-augmented-wave (PAW) method and found a pressure dependence on the bulk modulus of silica. ${ }^{[43]}$ Experimental measurements of silver nanowires loaded in tension along the $\left[\begin{array}{lll}0 & 1 & 1\end{array}\right]$ direction found a stiffening behavior. ${ }^{[44]}$ Copper nanowires also show either stiffening or softening, depending on the crystallographic orientation, as shown by earlier analyses using the EAM potential. ${ }^{45]}$

In order to gain a deeper understanding of the corresponding mechanisms that explain the sizedependent behavior of the material, we now proceed to analyze the stress fields during deformation. We first turn to the failure mechanisms found in the silica foil structures displayed in Figure 4. We find that void

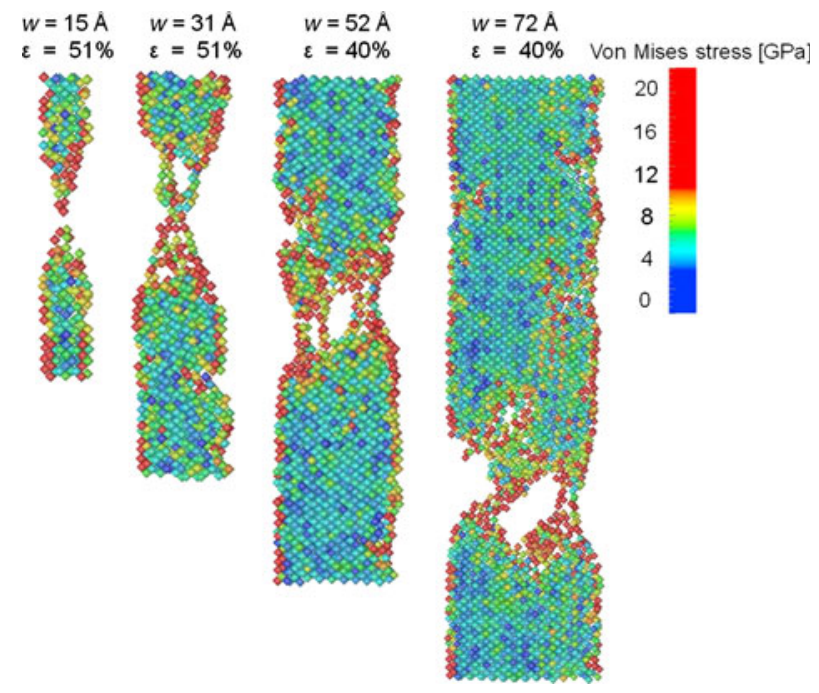

Fig. 4-Von Mises stress field during failure, for different foil wall widths (the strain value at which the snapshot was taken is indicated in the plot). The highest stresses are localized near the failure zones and the surface. The figure also reveals the deformation mechanisms, showing the locations of void nucleation and coalescence. Bifurcation of voided regions and shearlike behavior is observed for $w=72 \AA$. A beading mechanism narrowing down to thin strings of atoms is observed for the final stage in deformation. In order to improve image clarity, we only show the stress values associated with silicon atoms within the silica system. 
formation occurs for $w>15 \AA$, and first nucleates near the surfaces. Subsequently, voids coalesce and grow, ultimately leading to fracture. The trajectory of void nucleation events occurs roughly on one plane inclined to the loading axis, and for the largest $w=72 \AA$ structure, they bifurcate onto two planes, allowing for larger regions of void coalescence. In all silica foil structures, beading down to thin atomic chains is observed toward the end stages of failure before complete fracture is observed. Although no clear signs of dislocation or shear band nucleation are observed, the initial mechanisms of cavity nucleation are consistent with other MD simulations and experimental studies of silica deformation. ${ }^{[30,46]}$ Previous studies on small diameter nanowires (similar in size to those in our study) have determined that the mechanism of yielding by dislocation is absent, and demonstrated that the main mechanical instability is a disorder-order transformation, resulting in the reduction in size of the nanowire neck until single atom chains emerge. ${ }^{[24,47]}$

We proceed with analysis of the silica meshes, as shown in Figure 5, which shows the equivalent von Mises stress field at the maximum stress. For larger wall widths, high stress is mostly concentrated on the surface and specifically near the edges, thus suggesting possible locations for crack or shear nucleation. However, with lower wall widths, the stresses become relatively homogeneous throughout the structure. For large deformation, the void shape gradually changes from a rectangular to a hexagonal one for decreasing wall widths, and can be clearly seen for $w \leq 31 \AA$ A. High levels

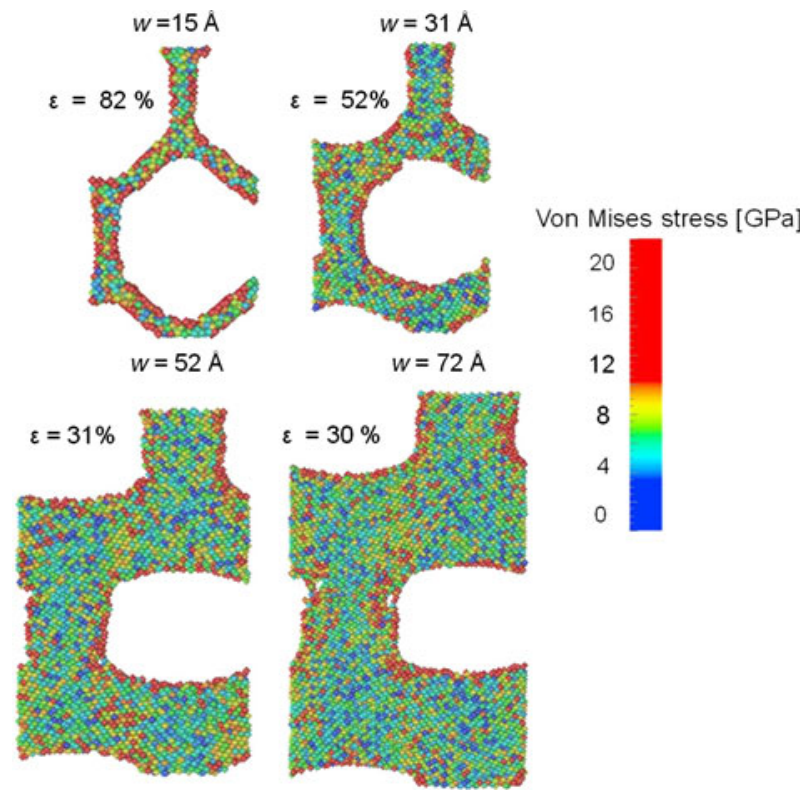

Fig. 5-Von Mises stress field at the maximum stress, for different mesh wall widths (the strain value at which the snapshot was taken is indicated in the plot). For widths smaller than $\approx 31 \AA$, the structure at the maximum stress becomes hexagonal, and the stress is distributed homogeneously throughout the structure. For larger wall widths, high stresses are concentrated around the corners. Moreover, the initial, rectangular shape of the structure is maintained. In order to improve image clarity, we only show the stress values associated with silicon atoms within the silica system.

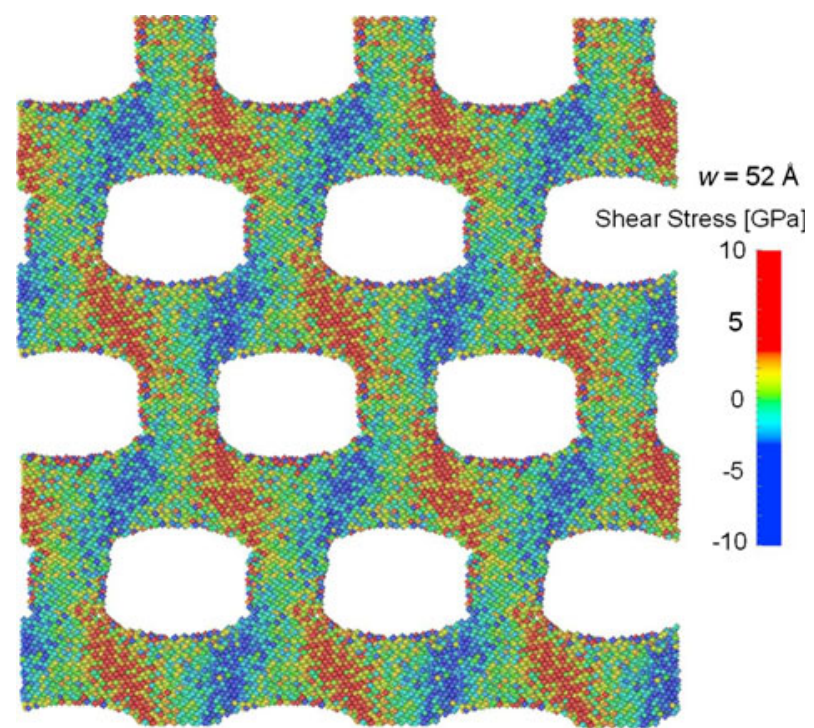

Fig. 6 - Shear stress $\sigma_{x y}$ taken at maximum stress for the system with wall width $w=52 \AA$. High regions of shear stress form a diagonal pattern and suggest possible areas where deformation occurs. We show multiple sets of the periodic cell so that the stress pattern can be clearly seen. In order to improve image clarity, we only show the stress values associated with silicon atoms within the silica system.
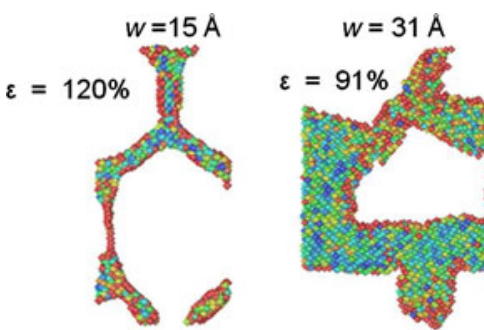

Von Mises stress [GPa]
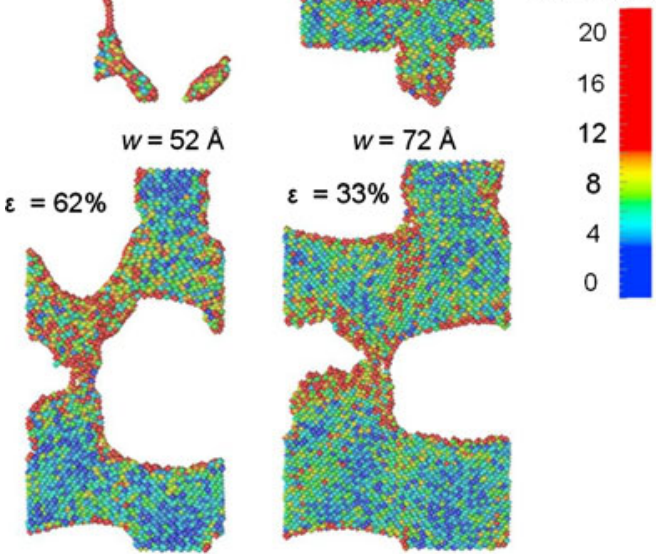

Fig. 7-Von Mises stress field during failure for different mesh wall widths (the strain value at which the snapshot was taken is indicated in the plot). For the systems with wall widths of 15 and $31 \AA$, necking and formation of beaded molecular structure are observed. At widths of $52 \AA$ and larger, cracks initiate from the corners. For $w \geq$ $31 \AA$, we observe the formation of voids within the sample, specifically within the regions surrounding the failure process zone. The failure mechanism remains similar for $w \leq 52 \AA$ and is characterized by a structural change from a rectangular to a hexagonal shape. An analogy to deformation in mascroscopic plastic hinges can be drawn to describe the mechanism for accommodating large deformations. For larger systems, however, the failure mode is consistently crack propagation, an effect that is confirmed to exist for varying strain rates. In order to improve image clarity, we only show the stress values associated with silicon atoms within the silica system. 
of shear stress also manifest in a diagonal pattern, as shown in Figure 6 for $w>31 \AA$, which facilitate possible regions of void nucleation and the start of failure. Once crack failure starts, the stress is concentrated around the fracture process zone, as shown in Figure 7. The deformation mechanisms observed for varying wall widths are dramatic in that they correlate with the peaking of toughness and can be summarized as follows. For $w>62 \AA$ and $w<21 \AA$, the dominant failure mechanisms are brittle crack propagation and beading down to thin atomic chains, respectively. However, for $21 \leq w \leq 62 \AA$, crack propagation and shear mechanisms occur in a competing fashion and allow for increased toughness. For example, for the $w=52 \AA$ structure, we observe a crack arrest phenomenon and corresponding increase of tensile stress from 2 to $2.8 \mathrm{GPa}$, which is due to shear mechanisms occurring within the junctions of the mesh elsewhere (Figure 8).

The analysis discussed in the preceding paragraph explains the remarkable stress-strain response of mesh structures with thin wall widths, as shown in Figure 3(b). The key to explain these is the geometric pattern that allows large deformations to be accommodated by the mesh by changing from a rectangular pattern to a hexagonal one at large strains (e.g., in Figures 5 and 7), specifically for wall widths below 31 A. The fundamental reason for these very large strains without failure is due to the more homogeneous distribution of stresses and the geometry transformation from rectangular to a hexagonal shape for smaller wall widths.

In Figure 9, we summarize the effect of wall width variations and the hierarchy level on the mechanical properties - the plastic regime, toughness, maximum stress, and ductility. In all structures considered here, the plastic regime increases with decreasing $w$. The maximum stress and modulus both increase with the wall width, and the ductility increases for smaller wall widths. For the largest wall width in silica and silicon structures, the range of ductility is between $\approx 30$ and 50 pct. The greatest ductility is observed for the silica mesh with smallest wall width of $5 \AA$, reaching 120 pct. The silica foil shows a gradual increase in modulus with width, with a maximum of $6.7 \mathrm{GPa}$ for $w=72 \AA$, whereas the mesh structures sharply increase in modulus,

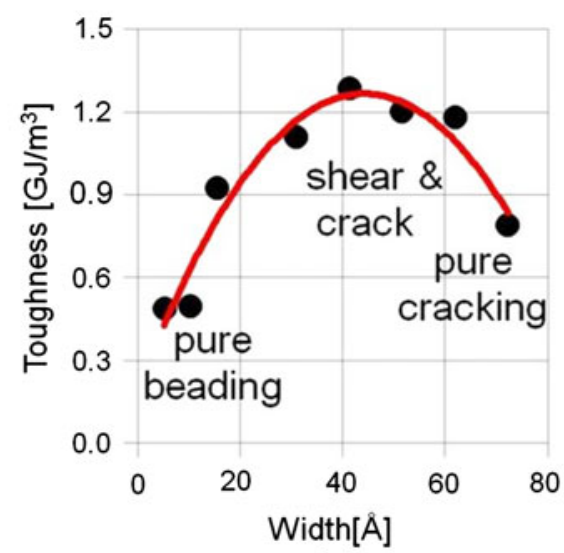

(a)

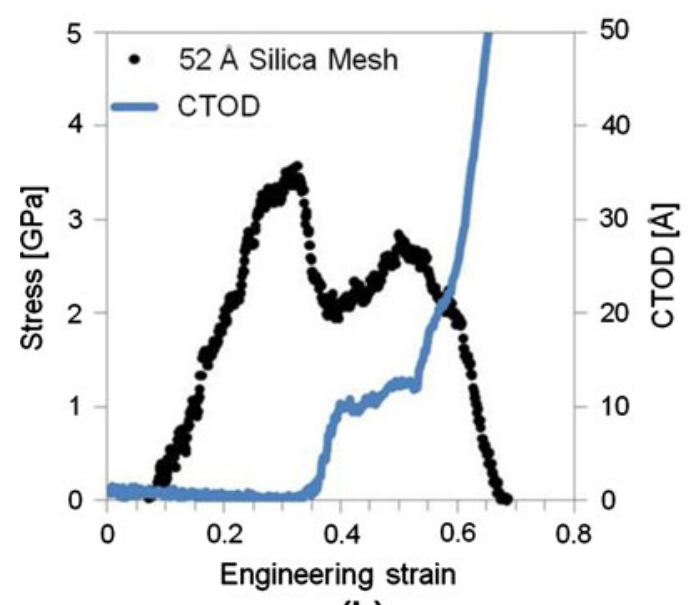

(b)

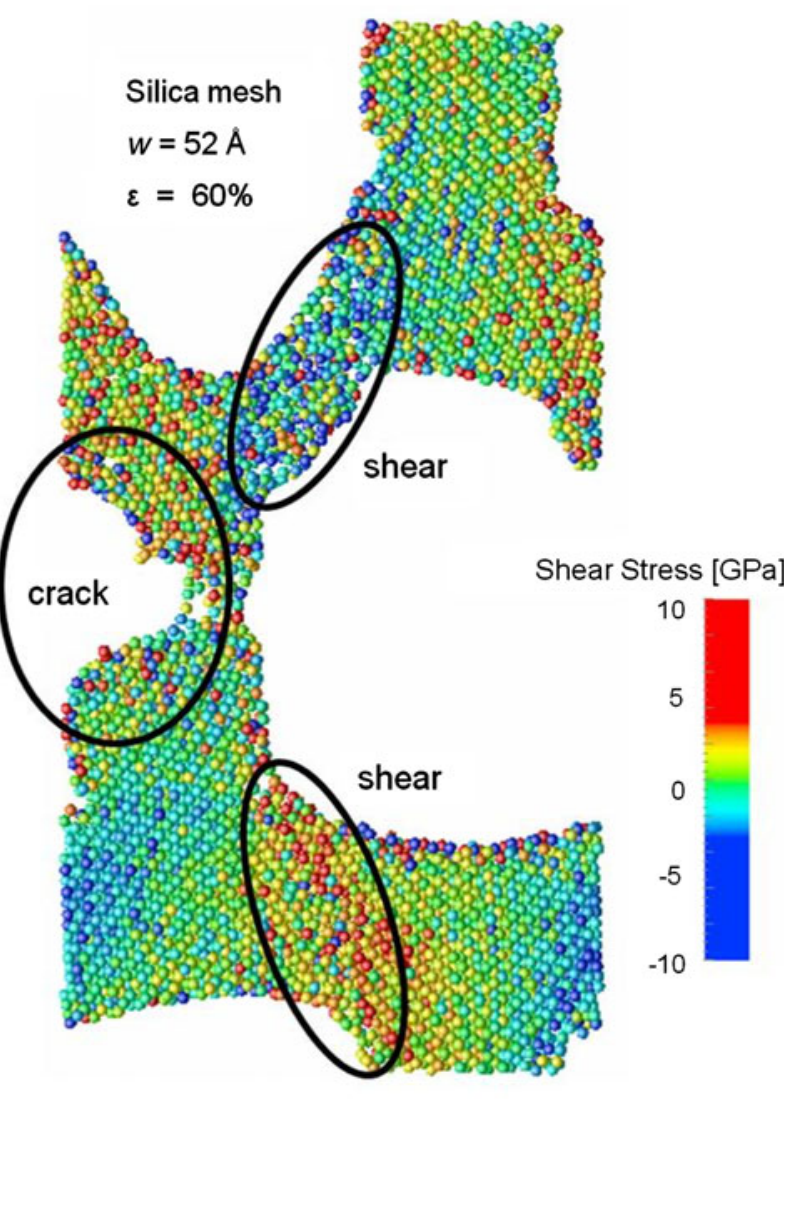

(c)

Fig. 8-Panel (a): Toughness map with corresponding failure mechanism for the silica mesh. Panel (b): Toughening and stiffening mechanisms are caused by competing mechanisms of shear and crack formation. The crack tip opening displacement (CTOD) measurement reveals crack arrest and is plotted against the corresponding stress-strain data, which reveal how well correlated both mechanisms are. Panel (c): Locations of shear and crack formation. For purposes of clarity, only the silicon atoms are shown. 


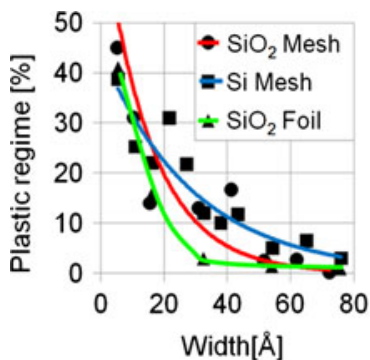

(a)

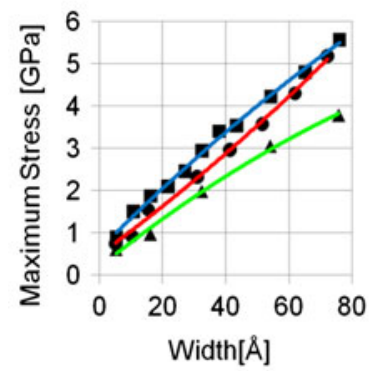

(c)

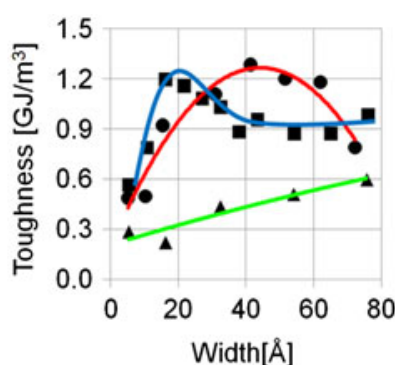

(b)

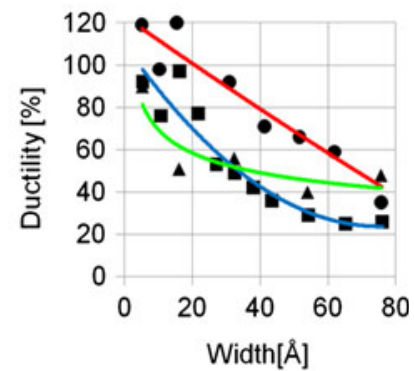

(d)
Fig. 9-Comparison between nanoporous silica, nanoporous silicon, and silica foil structures showing the effect of wall width on $(a)$ the plastic regime, (b) toughness, (c) maximum stress, and (d) ductility. Data for the nanoporous silicon is retrieved from Ref. 22. Both the maximum stress and modulus are found to increase with the wall width. The ductility generally increases for smaller wall widths. The plastic regime is estimated by measuring the length of the linear plateau region, which is associated with constant stress. In silicon, for wall widths larger than $27 \AA$, the toughness plateaus are at or around $9 \times 10^{8} \mathrm{~J} / \mathrm{m}^{3}$. Between 16 and $27 \AA$, a sharp increase in toughness is observed, with a maximum at $\approx 16 \AA$. Below $16 \AA$, the toughness drops to around $7 \times 10^{8} \mathrm{~J} / \mathrm{m}^{3}$, denoting an inverse trend. In the silica mesh, the highest toughness is observed for wall widths of $41 \AA$, reaching values $\approx 1.29 \times 10^{9} \mathrm{~J} / \mathrm{m}^{3}$. The silica foil generally increases in toughness with the wall width, yet has lower toughness when compared to the mesh structures. Thus, by increasing the level of hierarchy, a higher toughness, maximum stress, and modulus can be achieved.

reaching 36 and $29 \mathrm{GPa}$ for silicon and silica meshes, respectively. The effect of hierarchy on toughness is quite striking because the foil does not show a sizedependent toughness peaking response, as was observed for the meshes, and because it has a consistently lower toughness than the meshes. For example, the maximum toughnesses observed in silicon and silica meshes are $1.20 \times 10^{9}$ and $1.29 \times 10^{9} \mathrm{~J} / \mathrm{m}^{3}$, respectively, yet the silica foil reaches only $0.60 \times 10^{9} \mathrm{~J} / \mathrm{m}^{3}$. The reason for greater toughness in the higher hierarchy of meshes lies in competing mechanisms of shear and crack, wherein crack arrest is achieved either through shearing of another foil subcomponent in the mesh structure (as observed in the silica mesh, $w=52 \AA$ ) or through simultaneous cracking of different regions (as observed in the silicon mesh, $w=43 \AA$ ). These competing mechanisms are enabled through the hierarchical assembly of the foil elements into the mesh structure and could not be achieved in unit foil structures alone. This result demonstrates that including higher levels of hierarchy is beneficial in improving the mechanical properties and deformability of silica structures.

\section{CONCLUSIONS}

By using an atomistic simulation approach based on the first principles reactive force field ReaxFF, we investigated the impact of hierarchical structures on the mechanical response of the most abundant mineral on earth, silica. By incorporating a hierarchical design concept inspired by diatoms algae, we modeled two levels of geometric hierarchies: (1) a nanoscale foil of silica and (2) a nanoscale silica mesh composed of interlocking foils. These arrangements were then studied with different wall widths in order to reveal the effect of size scaling on their mechanical properties. Next, we presented a comparison between the mechanical properties of silica meshes and silica foils. Our findings suggest that higher levels of hierarchy are critical to greatly improving toughness and can increase toughness by up to 200 pet from silica foil to silica mesh.

We ascribe these magnificent improvements in mechanical properties of the mesh structures from two competing atomistic mechanisms of deformation: shear and brittle crack propagation. In the toughest silica mesh, for example, a crack arrests due to shearing of another strut, which consequently stiffens the system (Figure 8). Interestingly, a size-dependent peaking of toughness was observed for the $\mathrm{Si}$ and $\mathrm{SiO}_{2}$ meshes, with a maximum toughness of $1.29 \times 10^{9} \mathrm{~J} / \mathrm{m}^{3}$ corresponding to $w=41 \mathrm{~A}$ for the $\mathrm{SiO}_{2}$ mesh (Figure 9(b)). The increased toughness of these nanostructures also makes them viable candidates for impact-resistant lightweight structures, and this should be tested in further detail. Another powerful concept derived from this study is the ability to transform a brittle material into a ductile one by simply manipulating the geometry of a constituent structure to resemble that of ordered nanopores, or mesh. The reasons for the high ductility are threefold: (1) a homogeneous distribution of surface stress throughout the entire structure, (2) a conformational change from rectangular to hexagonal pores, and (3) competing mechanisms of shear and crack arrest.

In a similar fashion to structural materials found in nature, such as bone, nacre, or diatom shells, it seems that hierarchy is a cornerstone of nanomaterial design for superior mechanical properties. More importantly, the concept of hierarchical structures made of the same materials (as demonstrated in this article) is fundamental in order to fully realize the enormous potential of nanomaterial design. To the best of the authors' knowledge, this article is the first to elucidate, at the atomistic scale and with near QM accuracy, the complex mechanical response and failure mechanisms due to implementing hierarchy in silica. The key contribution of this work is that, by introducing structural hierarchies, a weakness can be turned to strength, that is, an intrinsically strong but brittle material becomes exceedingly tough, strong, and ductile. The fact that a similar behavior was found in silicon ${ }^{[22]}$ suggests that this may indeed be a generic design concept that could be used for many materials. The observation that a weakness is turned into strength is also reminiscent of recent findings of similar behaviors of $\mathrm{H}$ bonds, which are by themselves also highly brittle, weak elements but reach 
extreme levels of toughness and strength once arranged in particular hierarchical patterns. ${ }^{[48]}$

Future research lies in addressing the impact of a larger number of hierarchy levels on the mechanical properties of silica nanostructures and the effect of water and hydrogen termination on hierarchical silica. Interestingly, a previous study used the semiempirical QM methods, PM3, and PM5 along with the BKS potential and found that water reduces the critical stress and failure strain of silica nanorods, ${ }^{[42]}$ and it is expected that similar effects might be crucial for the system considered here as well.

\section{ACKNOWLEDGMENTS}

We acknowledge support from the Army Research Office (Grant No. W9-11NNF-06-1029), the National Science Foundation through a Graduate Research Fellowship, the Gates Millennium Scholars Program, and a graduate research fellowship awarded by the Department of Civil and Environmental Engineering at the Massachusetts Institute of Technology.

\section{REFERENCES}

1. J. Aizenberg, J.C. Weaver, M.S. Thanawala, V.C. Sundar, D.E. Morse, and P. Fratzl: Science, 2005, vol. 309 (5732), pp. 275-78.

2. P. Fratzl and R. Weinkamer: Prog. Mater. Sci., 2007, vol. 52, pp. 1263-334.

3. R.O. Ritchie, M.J. Buehler, and P. Hansma: Phys. Today, 2009, vol. 62 (6), pp. 41-47.

4. M.J. Buehler and Y.C. Yung: Nat. Mater., 2009, vol. 8 (3), pp. $175-88$.

5. F.E. Round, R.M. Crawford, and D.G. Mann: Diatoms: Biology and Morphology of the Genera, Cambridge University Press, Cambridge, United Kingdom, 1990.

6. M. Hildebrand: Chem. Rev., 2008, vol. 108 (11), pp. 4855-74.

7. J.A. Raven and A.M. Waite: Tansley Rev., 2003, vol. 162, pp. 45-61.

8. C.E. Hamm and V. Smetacek: in Evolution of Primary Producers in the Sea, P.G. Falkowski and A.H. Knoll, eds., Elsevier, Boston, MA, 2007, pp. 311-32.

9. S.W. Fowler and N.S. Fisher: Deep Sea Res., 1983, vol. 39 (9), pp. 963-69.

10. H.-C. Shin, J.A. Corno, J.L. Gole, and M. Liu: J. Power Sources, 2005, vol. 139 (1-2), pp. 314-20.

11. E.K. Prince, T.L. Myers, and J. Kubanek: Limnol. Oceanogr., 2008, vol. 53 (2), pp. 531-41.

12. Z. Bao, M.R. Weatherspoon, S. Shian, Y. Cai, P.D. Graham, S.M. Allan, G. Ahmad, M.B. Dickerson, B.C. Church, Z. Kang, H.W. Abernathy Iii, C.J. Summers, M. Liu, and K.H. Sandhage: Nature, 2007, vol. 446 (7132), pp. 172-75.

13. D. Losic, J.G. Mitchell, and N.H. Voelcker: Adv. Mater., 2009, vol. 21 (29), pp. 2947-58.

14. N. Tokranova, I.A. Levitsky, B. Xu, J. Castracane, and W.B. Euler: Hybrid Solar Cells Based on Organic Material Embedded into Porous Silicon, SPIE, San Jose, CA, 2005.

15. C. Jeffryes, T. Gutu, J. Jiao, and G.L. Rorrer: ACS Nano, 2008, vol. 2 (10), pp. 2103-12.

16. W. Fenical: Plants: the Potentials for Extracting Protein, Medicines, and Other Useful Chemicals: Workshop Proc., Washington, DC, U.S. Government Printing Office, Washington, DC, 1983, pp. $147-53$
17. J.T. Allen, L. Brown, R. Sanders, C. Mark Moore, A. Mustard, S. Fielding, M. Lucas, M. Rixen, G. Savidge, S. Henson, and D. Mayor: Nature, 2005, vol. 437 (7059), pp. 728-32.

18. E. Litchman, C.A. Klausmeier, and K. Yoshiyama: Proc. Nat. Acad. Sci., 2009, vol. 106 (8), pp. 2665-70.

19. C.E. Hamm, R. Merkel, O. Springer, P. Jurkojc, C. Maier, K. Prechtel, and V. Smetacek: Nature, 2003, vol. 421 (6925), pp. $841-43$.

20. D. Losic, K. Short, J.G. Mitchell, R. Lal, and N.H. Voelcker: Langmuir, 2007, vol. 23 (9), pp. 5014-21.

21. D. Losic, R.J. Pillar, T. Dilger, J.G. Mitchell, and N.H. Voelcker: J. Porous Mater., 2007, vol. 14, pp. 61-69.

22. A.P. Garcia and M.J. Buehler: Comput. Mater. Sci., 2010, vol. 48 (2), pp. 303-09.

23. H.S. Park: Nanotechnology, 2009, vol. 20 (11), p. 115701.

24. T.Y. Kim, S.S. Han, and H.M. Lee: Mater. Trans., 2004, vol. 45 (5), pp. 1442-49.

25. T.J. Chuang, P.M. Anderson, M.K. Wu, S. Hsieh, and T.-j. Chuang: in Nanomechanics of Materials and Structures, Springer, Netherlands, 2006, pp. 67-78.

26. T. Namazu and Y. Isono: Sens. Actuat. A, Phys., 2003, vol. 104 (1), pp. 78-85.

27. H. Ni, X. Li, and H. Gao: Appl. Phys. Lett., 2006, vol. 88 (4), p. 043108 .

28. E.C. Silva, L. Tong, S. Yip, and K.J. Van Vliet: Size Effects on the Stiffness of Silica Nanowires, Small, 2006, vol. 2 (2), pp. 239-43.

29. J.P Lucas, N.R. Moody, S.L. Robinson, J. Hanrock, and R.Q. Hwang: Scripta Metall. Mater., 1995, vol. 32 (5), pp. 743-48.

30. D. Bonamy, S. Prades, C. Rountree, L. Ponson, D. Dalmas, E. Bouchaud, K. Ravi-Chandar, and C. Guillot: Int. J. Fract., 2006, vol. 140 (1), pp. 3-14.

31. M.J. Buehler, H. Tang, A.C.T. van Duin, and W.A. Goddard: Phys. Rev. Lett., 2007, vol. 99, p. 165502.

32. M.J. Buehler, A.C.T. van Duin, and W.A. Goddard: Phys. Rev. Lett., 2006, vol. 96 (9), p. 095505.

33. J.C. Fogarty, H.M. Aktulga, A.Y. Grama, A.C.T. van Duin, and S.A. Pandit: J. Chem. Phys., 2010, vol. 132 (17), pp. 174704-10.

34. A.C.T. van Duin, S. Dasgupta, F. Lorant, and W.A. Goddard: J. Phys. Chem. A, 2001, vol. 105, p. 9396.

35. A.C.T. van Duin, A. Strachan, S. Stewman, Q. Zhang, X. Xu, and W.A. Goddard: J. Phys. Chem. A, 2003, vol. 107 (19), pp. 3803-11.

36. W.W. Zhang, Q.A. Huang, H. Yu, and L.B. Lu: in Micro and Nano Technology-1st Int. Conf. of Chinese Society of Micro/Nano Technology (Csmnt), X. Wang, ed., Trans Tech Publications Ltd., Stafa-Zurich, 2009, pp. 315-19.

37. D. Raymand, A.C.T. van Duin, M. Baudin, and K. Hermansson: Surf. Sci., 2008, vol. 602 (5), pp. 1020-31.

38. W. Liu, K. Zhang, H. Xiao, L. Meng, J. Li, G.M. Stocks, and J. Zhong: Nanotechnology, 2007, vol. 18 (21), p. 215703.

39. H.J.C. Berendsen, J.P.M. Postma, W.F. van Gunsteren, A. DiNola, and J.R. Haak: J. Chem. Phys., 1984, vol. 81 (8), pp. 3684-90.

40. J.A. Zimmerman, E.B. Webb III, J.J. Hoyt, R.E. Jones, P.A. Klein, and D.J. Bammann: Model. Sim. Mater. Sci. Eng., 2004, vol. 12, pp. S319-S332.

41. T. Zhu, J. Li, S. Yip, R.J. Bartlett, S.B. Trickey, and N.H. de Leeuw: Molec. Simul., 2003, vol. 29 (10), pp. 671-76.

42. E. Silva, J. Li, D. Liao, S. Subramanian, T. Zhu, and S. Yip: J. Comput. Aided Mater. Des., 2006, vol. 13 (1), pp. 135-59.

43. H. Kimizuka, S. Ogata, and Y. Shibutani: Phys. Status Solidi (b), 2007, vol. 244 (3), pp. 900-09.

44. B. Wu, A. Heidelberg, J.J. Boland, J.E. Sader, X.M. Sun, and Y.D. Li: Nano Lett., 2006, vol. 6 (3), pp. 468-72.

45. H. Liang, M. Upmanyu, and H. Huang: Phys. Rev. B, 2005, vol. 71 (24), p. 241403.

46. C.L. Rountree, S. Prades, D. Bonamy, E. Bouchaud, R. Kalia, and C. Guillot: J. Alloys Compd., 2007, vols. 434-435, pp. 60-63.

47. H. Mehrez and S. Ciraci: Phys. Rev. B, 1997, vol. 56 (19), p. 12632.

48. S. Keten, Z. Xu, B. Ihle, and M.J. Buehler: Nat. Mater., 2010, vol. 9, pp. 359-67.

49. N. Kroger: Curr. Opin. Chem. Biol., 2007, vol. 11 (6), pp. 662-69.

50. N. Lundholm, Ø. Moestrup, G.R. Hasle, and K. Hoef-Emden: J. Phycol., 2003, vol. 39 (4), pp. 797-813. 